\title{
SUMS OF IRREDUCIBLE OPERATORS ${ }^{1}$
}

PETER A. FILLMORE AND DAVID M. TOPPING

Let $\mathfrak{T}$ be a separable, infinite-dimensional complex Hilbert space, and let $B(\mathcal{H C})$ denote the algebra of all bounded linear operators on $\mathcal{H C}$. A (closed, linear) subspace $\mathfrak{T C}$ of $\mathfrak{H C}$ is said to reduce an operator

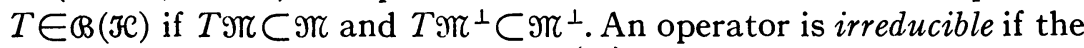
only subspaces which reduce it are $\{0\}$ and $\Re$. Halmos has recently shown that the irreducible operators are dense in $B(\mathfrak{F C})$ in the norm topology [3]. Our purpose here is to note that irreducible operators abound in another sense.

THEOREM 1. Every operator in $B(\mathfrak{H C})$ is the sum of four irreducible operators.

This result will be proved by means of

Theorem 2. A selfadjoint operator is the real part of an irreducible operator if and only if it is not a scalar.

We begin with the following lemma.

Lemma. Each projection other than 0 and $I$ is the real part of an irreducible operator.

Write $\mathfrak{K}=\mathfrak{K} \oplus \mathcal{K}$, let $\left\{e_{n} \mid n=1,2, \cdots\right\}$ and $\left\{f_{n} \mid n=1,2, \cdots\right\}$ be orthonormal bases of $\mathscr{K}$ with

$$
f_{1}=\frac{\sqrt{ } 6}{\pi} \sum_{n=1}^{\infty} \frac{1}{n} e_{n},
$$

and let $S$ be the shift operator on $\mathscr{K}$ defined by $S f_{n}=f_{n+1}$. With $A=S / \sqrt{ } 2$, the operator

$$
U=\left(\begin{array}{cc}
A & \left(I-A A^{*}\right)^{\frac{3}{3}} \\
\left(I-A^{*} A\right)^{\frac{3}{3}} & -A^{*}
\end{array}\right)
$$

on $\mathfrak{H C}$ is unitary [2, Problem 177], and

$$
U\left(\begin{array}{ll}
I & 0 \\
0 & 0
\end{array}\right) U^{*}=\frac{1}{2}\left(\begin{array}{cc}
S S^{*} & S \\
S^{*} & I
\end{array}\right) .
$$

Therefore, the right side of this equation is a projection $P$ of infinite rank and infinite nullity.

Received by the editors September 29, 1967.

${ }^{1}$ Research supported in part by grants from the National Science Foundation. 
Now let $K=A \oplus B$, where the selfadjoint operators $A$ and $B$ are defined on $\mathcal{K}$ by $A e_{n}=e_{n} / n$ and $B e_{n}=e_{n} / n \pi$. We show that $P+i K$ is irreducible, which will prove the lemma for projections of infinite rank and nullity. To this end, let

$$
\left(\begin{array}{ll}
X & Y \\
Y^{*} & Z
\end{array}\right)
$$

be a projection which commutes with $P+i K$, or equivalently with $P$ and $K$. The commutativity with $K$ gives $A X=X A, B Z=Z B$, and $A Y=Y B$, so $A Y e_{n}=Y B e_{n}=Y e_{n} / n \pi, Y e_{n}=0$ for all $n$, and therefore $Y=0$. Further, the equations $A X=X A$ and $B Z=Z B$ imply that $X$ and $Z$ are diagonal in the basis $\left\{e_{n}\right\}$ (that is, each $e_{n}$ is an eigenvector for $X$ and $Z$ ). Next, the commutativity with $P$ gives $X S S^{*}=S S^{*} X$ and $X S=S Z$. Since $S S^{*}$ is the projection on the span of the $f_{n}$ for $n \geqq 2$, the first equation shows that $f_{1}$ is an eigenvector for $X$. Coupled with the fact that $X$ is diagonal in the basis $\left\{e_{n}\right\}$, this shows that $X$ is scalar. Then $X S=S Z$ implies $Z=S^{*} X S=X S^{*} S=X$. Consequently the projection

$$
\left(\begin{array}{ll}
X & Y \\
Y^{*} & Z
\end{array}\right)
$$

is scalar, and must therefore be 0 or $I$.

We have not been able to find a proof of this type for projections of finite (or cofinite) rank. Before proceeding with this case, we wish to note that with the information at hand, we can prove a weakened form of Theorem 1, namely: every operator is the sum of a finite number of irreducible operators. Since every operator is a finite linear combination of projections [1], and since a projection is either the sum or the difference of two projections of infinite rank and nullity, it suffices to consider projections of infinite rank and nullity. For such a projection $P$, there is a selfadjoint operator $K$ such that $P+i K$ is irreducible. Then $P=\frac{1}{2}(P+i K)+\frac{1}{2}(P-i K)$, and the operators $\frac{1}{2}(P \pm i K)$ are irreducible.

Returning to the proof of the lemma, let $\mathfrak{H C}=L^{2}(\mu)$, with $\mu$ Lebesgue measure on $[0,1]$, and let $P$ be the projection on the polynomials of degree at most $n-1$. Then $P$ is of rank $n$, and we shall show that $P+i K$ is irreducible, where $K$ is defined by $(K f)(t)=t f(t)$ for all $f \in L^{2}$. If $E$ is a projection which commutes with $K$, then $E$ is given by multiplication by the characteristic function $\phi$ of a measurable subset $A$ of [0,1] [2, Problem 115]. If $E$ also commutes with $P$, then $P$ leaves invariant the subspace of functions which vanish outside of $A$. 
In particular $P \phi$ vanishes outside of $A$, and since it is a polynomial, we have either $\mu(A)=1$ or $P \phi=0$. In the former case $E=I$, and in the latter $\phi$ is orthogonal to the function identically equal to 1 , so $\mu(A)=0$ and $E=0$. Therefore $P+i K$ is irreducible. Since $(I-P)+i K$ is also irreducible, this completes the proof of the lemma.

We remark that a proof along these lines may be constructed for the first part of the lemma. Let $\mathfrak{F}=L^{2}(0,2 \pi)$, let $\left\{e_{n}\right\}$ be the usual basis of exponentials, let $K$ be defined by $K e_{n}=e_{n} / n$ for $n \neq 0$ and $K e_{0}=0$, and let $P$ be the projection on $L^{2}(0,2 \pi a)$ with $a$ irrational and $a \in(0,1)$. Then $P+i K$ is irreducible. The proof is similar to the above and will be omitted.

For the proof of Theorem 2, let $H$ be a nonscalar selfadjoint operator. Then there exists a spectral projection $P$ of $H$ distinct from 0 and $I$. By the lemma there is a selfadjoint operator $K$ such that $P+i K$ is irreducible. Since for any operator $T, T H=H T$ implies $T P=P T$, it follows that $H+i K$ is irreducible. The converse is obvious: an operator with scalar real part is normal and therefore reducible.

Finally we note that any selfadjoint operator is the sum of two irreducible operators, from which Theorem 1 follows. If $H$ is nonscalar this is a consequence of Theorem 2. If $H=\alpha I$ and $T$ is any irreducible operator, then $\alpha I-T$ is irreducible and $\alpha I=T+(\alpha I-T)$.

ADDED IN PROOF. Heydar Radjavi has recently improved the number four in Theorem 1 to two, which is best possible. His result will appear in these Proceedings.

\section{REFERENCES}

1. P. A. Fillmore, Sums of operators with square zero, Acta Sci. Math. (Szeged) 28 (1967), 285-288.

2. P. R. Halmos, A Hilbert space problem book, Van Nostrand, Princeton, N. J., 1967.

3. - Irreducible operators, Michigan Math. J. 15 (1968), 215-223.

INDIANA UNIVERSITY AND

TULANE UNIVERSITY 